\title{
Few-body approach to diffraction of small helium clusters by nanostructures
}

\author{
Gerhard C. Hegerfeldt? \\ Institut für Theoretische Physik, Universität Göttingen \\ Bunsenstr. 9, 37073 Göttingen, Germany \\ and \\ Thorsten Köhler| \\ Max-Planck-Institut für Strömungsforschung \\ Bunsenstr. 10, D-37073 Göttingen, Germany
}

\begin{abstract}
We use few-body methods to investigate the diffraction of weakly bound systems by a transmission grating. For helium dimers, $\mathrm{He}_{2}$, we obtain explicit expressions for the transition amplitude in the elastic channel.
\end{abstract}

\section{1. Introduction}

In recent years typical optics experiments have been carried over to atoms, among them diffraction by double slits [1] and by transmission gratings which were produced by nanostructure techniques [2, 3]. The usual wave-theoretical methods of classical optics (Huygens, Kirchhoff) seem to give excellent descriptions of these experiments [4] so that apparently not much more than a good textbook on classical optics seems to be needed.

This simple picture, however, has changed. Recently Schöllkopf and Toennies [2, 5, 6] have performed impressive diffraction experiments with helium dimers and helium clusters consisting of up to 26 atoms. A typical diffraction pattern is shown in Fig. 1. The helium dimer $\mathrm{He}_{2}$, discovered a few years ago [8, 2], has an extremely low binding energy of $-0.11 \mu \mathrm{eV}$ [7], with no excited states. The binding energy is much smaller than the incident kinetic energy of typical experiments. Its diameter is estimated to be about $6 \mathrm{~nm}$ [9]. Present day slit widths are as low as $50 \mathrm{~nm}$ and a further reduction is expected.

When a system is observed at a nonzero diffraction angle it clearly has received a lateral moment transfer from the grating. For weakly bound systems like $\mathrm{He}_{2}$ or higher clusters this might induce breakup processes which in turn might change the diffraction pattern. Similarly, the size of the bound system may have an influence. If so, what will be the effect? Will the diffraction pattern change

\footnotetext{
${ }^{1}$ Invited talk delivered at the 16TH EUROPEAN CONFERENCE ON FEW-BODY PROBLEMS IN PHYSICS, Autrans, June 1-6, 1998

2 e-mail: hegerf@theorie.physik.uni-goettingen.de

${ }^{3}$ e-mail: koehler@theorie.physik.uni-goettingen.de
} 
drastically? Will the diffraction peaks still be at the same locations as for point particles? These questions will be investigated and answered in the following, with emphasis on $\mathrm{He}_{2}$. More technical details can be found in Ref. [10.

Diffraction by a transmission grating really means scattering by the bars of the grating, and to include breakup processes and finite-size effects we use multichannel scattering theory. We describe the effect of the grating bars by means of a short-range repulsive ('reflective') potential. The possible role of attractive parts to the potential will be discussed at the end. As usual we neglect the electronic degrees of freedom.

We thus consider a weakly bound system of two particles and its scattering by an external potential. Due to the presence of the external potential this problem cannot be reduced to a one-particle problem but has more resemblance, at least mathematically, to a three-body problem. In fact, we will show that the Faddeev approach to the three-body problem in its formulation by Alt, Grassberger, and Sandhas [11] can be carried over and adapted to the two-body problem with external potential.

\section{AGS Equations for Two Particles with Ex- ternal Potential}

In this section we consider a bound two particle system ('molecule') scattered by a quite general obstacle. The coordinates are $\mathbf{x}_{1}$ and $\mathbf{x}_{2}$, the binding potential is $V\left(\mathbf{x}_{1}-\mathbf{x}_{2}\right)$, with negative binding energies $E_{\gamma}$ and bound states $\phi_{\gamma}(\mathbf{x})$. The external (obstacle) potential is

$$
W\left(\mathbf{x}_{1}, \mathbf{x}_{2}\right)=W_{1}\left(\mathbf{x}_{1}\right)+W_{2}\left(\mathbf{x}_{2}\right)
$$

where $W_{1}$ and $W_{2}$ are short-range and strongly repulsive, representing Dirichlet boundary conditions in the limit. For dimers one has $W_{1}=W_{2}$.

We first consider the process $\left|\mathbf{P}^{\prime}, \phi_{\gamma^{\prime}}\right\rangle \rightarrow\left|\mathbf{P}, \phi_{\gamma}\right\rangle$ from an incoming molecule of momentum $\mathbf{P}^{\prime}$ and internal state $\phi_{\gamma^{\prime}}$ to an outgoing molecule of momentum $\mathbf{P}$ and internal state $\phi_{\gamma}$, i.e. no breakup. We introduce the six-dimensional Green's operators $G_{0}(z)=\left(z-H_{0}\right)^{-1}, G(z)=\left(z-H_{0}-V-W\right)^{-1}, G_{V}(z)=\left(z-H_{0}-V\right)^{-1}$, and similarly $G_{W}(z)$, where $H_{0}$ is the free Hamiltonian (kinetic energy). One has the usual resolvent equations

$$
\begin{aligned}
& G=G_{V}+G_{V} W G \\
& G=G_{W}+G_{W} V G .
\end{aligned}
$$

The $T$ matrices for $V$ and $W$ are defined as

$$
\begin{aligned}
T_{V}(z) & \equiv V+V G_{V}(z) V \\
T_{W}(z) & \equiv W+W G_{W}(z) W .
\end{aligned}
$$




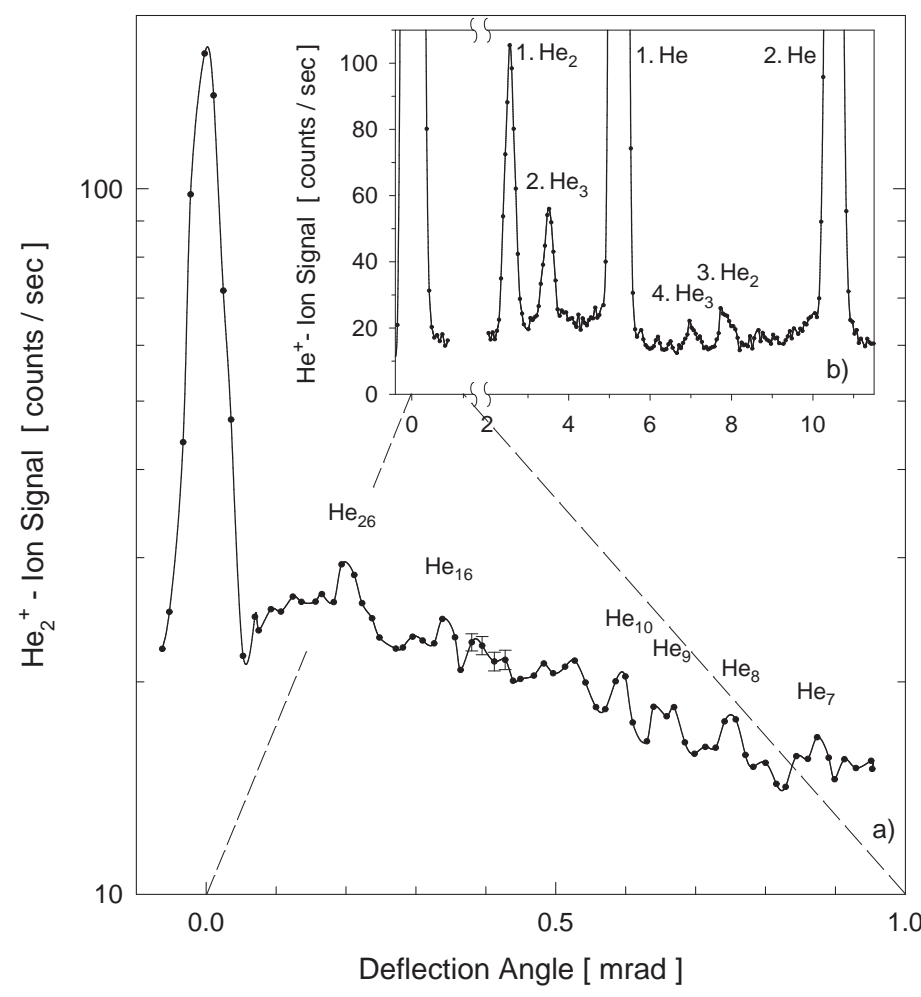

Figure 1: Diffraction of helium clusters for a source temperature of $6 \mathrm{~K}$ by a 100-nm-period transmission grating [6].

\section{Writing}

$$
T_{V}=V G_{V}\left(G_{V}^{-1}+V\right)=V G_{V} G_{0}^{-1}
$$

and similarly for $W$ gives

$$
\begin{gathered}
T_{V} G_{0}=V G_{V} \\
T_{W} G_{0}=W G_{W} .
\end{gathered}
$$

We now introduce operators $U_{V V}(z)$ and $U_{W V}(z)$ through

$$
\begin{array}{lll}
G(z) & = & G_{V}(z)+G_{V}(z) U_{V V}(z) G_{V}(z) \\
G(z) & = & G_{W}(z) U_{W V}(z) G_{V}(z) .
\end{array}
$$


As in Ref. [11] one easily shows that the transition amplitude for the above process is

$$
t\left(\mathbf{P}, \phi_{\gamma} ; \mathbf{P}^{\prime}, \phi_{\gamma^{\prime}}\right)=\left\langle\mathbf{P}, \phi_{\gamma}\left|U_{V V}(E+\mathrm{i} 0)\right| \mathbf{P}^{\prime}, \phi_{\gamma^{\prime}}\right\rangle .
$$

Equating Eqs. (2) and (7),

$$
G_{V}+G_{V} W G=G_{V}+G_{V} U_{V V} G_{V}
$$

and inserting Eq. (8) gives

$$
U_{V V}=W G_{W} U_{W V}=T_{W} G_{0} U_{W V}
$$

Similarly, equating Eqs. (3) and (8) and inserting Eq. (7) gives

$$
U_{W V}=G_{0}^{-1}+T_{V} G_{0} U_{V V}
$$

The last two equations are the AGS equations for two particles interacting through a potential $V$ and with an additional external potential $W$. These AGS equations decouple here, as seen by insertion of Eq. (12) into Eq. (11), which gives

$$
U_{V V}=T_{W}+T_{W} G_{0} T_{V} G_{0} U_{V V}
$$

This equation is exact and contains both breakup and finite-size effects. It lends itself to iteration, and to lowest order in $T_{V}$ one has

$$
U_{V V} \cong T_{W}
$$

By Eq. (9) this gives

$$
t\left(\mathbf{P}, \phi_{\gamma} ; \mathbf{P}^{\prime}, \phi_{\gamma^{\prime}}\right) \cong\left\langle\mathbf{P}, \phi_{\gamma}\left|T_{W}(E+\mathrm{i} 0)\right| \mathbf{P}^{\prime}, \phi_{\gamma^{\prime}}\right\rangle .
$$

Although $T_{W}$ is the transition operator for scattering of two asymptotically free particles by the external potential $W$ it is not on the energy shell in Eq. (15). Here the interaction $V$ is taken into account through the wave-functions $\phi_{\gamma}$ and $\phi_{\gamma^{\prime}}$.

Similarly to Eq. (9) one can show that

$$
U_{0 V} \equiv G_{0}^{-1}+T_{V} G_{0} U_{V V}+T_{W} G_{0} U_{W V}
$$

is the transition operator for breakup processes, and for this one only has to know $U_{V V}$ and $U_{W V}$. 


\section{Application to Diffraction of Helium Dimers}

The scattering amplitude in Eq. (15) can be evaluated in case of small diffraction angles for a transmission grating of $N$ bars of period $d$ and slit width $s$ [10]. We consider a bound system of two particles, each of mass $m$, and normal incidence. With $P$ the total momentum of the system and $P_{2}$ the lateral momentum transfer, the elastic scattering amplitude becomes of the form

$$
t\left(\mathbf{P}, \phi_{\gamma} ; \mathbf{P}^{\prime}, \phi_{\gamma}\right)=t_{\text {coh }}\left(P_{2}\right)+t_{\text {incoh }}\left(P_{2}\right) .
$$

The first term is a superposition of amplitudes from individual bars, while the second results from interactions with separate bars and it can be shown to be negligible if $d-s$ and $s$ are larger than the molecule. The coherent part is of the form

$$
t_{\mathrm{coh}}\left(P_{2}\right)=t_{\mathrm{bar}}^{\mathrm{mol}}\left(\gamma, P / M ; P_{2}\right) \frac{\sin \left(P_{2} N d / 2 \hbar\right)}{\sin \left(P_{2} d / 2 \hbar\right)} \delta\left(P_{3}\right)
$$

where the first factor is the molecular transition amplitude for a single bar of width $d-s$, for total momentum $P$ and total mass $M$. The second factor is the usual sharply peaked grating function known from optics, and it gives the diffraction peaks at the same locations as for point-particles with corresponding lateral momentum transfer. The $\delta$ function is due to the assumed infinite extent of the grating in the 3 direction. The amplitude of the diffraction peaks is determined

by $t_{\text {bar }}^{\text {mol }}$ The latter has been explicitly calculated by means of Eq. (15) in Ref. [10] and it depends only on the absolute value of the ground-state wave-function. One obtains

$$
\begin{aligned}
t_{\mathrm{bar}}^{\mathrm{mol}}(\gamma, P / M ; & \left.P_{2}\right)=-\frac{2 \mathrm{i} P}{(2 \pi)^{2} M} \frac{\sin \left[P_{2}(d-s) / 2 \hbar\right]}{P_{2}} \int \mathrm{d}^{3} x 2 \mathrm{e}^{\mathrm{i} P_{2} x_{2} / 2 \hbar}\left|\phi_{\gamma}(\mathbf{x})\right|^{2} \\
& +\frac{4 \mathrm{i} P}{(2 \pi)^{2} M} \int \mathrm{d} x_{1} \mathrm{~d} x_{3} \int_{0}^{d-s} \mathrm{~d} x_{2}\left|\phi_{\gamma}(\mathbf{x})\right|^{2} \sin \left[\frac{P_{2}}{2 \hbar}\left(d-s-x_{2}\right)\right] / P_{2} .
\end{aligned}
$$

The expression preceding the first integral is the single-bar amplitude for a point particle of mass $\mathrm{M}$ and total momentum $P$. The complete expression on the r.h.s. reduces to this if $\left|\phi_{\gamma}(\mathbf{x})\right|^{2}$ contracts to a point.

In Fig. 2 we have plotted $\left|t_{\mathrm{bar}}^{\mathrm{mol}}\right|^{2}$ for $\mathrm{He}_{2}$ and the corresponding quantity for a point particle. The bar width is $25 \mathrm{~nm}$. For a symmetric grating of period $50 \mathrm{~nm}$ the vertical lines in the inset indicate where the peaks of the grating functions cut out the diffraction peaks of first order, second order and so on. For a symmetric grating and point particles there are no even-order diffraction peaks since the single-bar amplitude vanishes there, as seen in the inset. For the dimer this is not true, and it therefore has small even-order peaks which become more pronounced for smaller bar and slit widths.

Intuitively we expect that finite-size effects of $\mathrm{He}_{2}$ might be partially taken into account by a larger bar and smaller slit width. Fig. 3 shows that the single-bar 


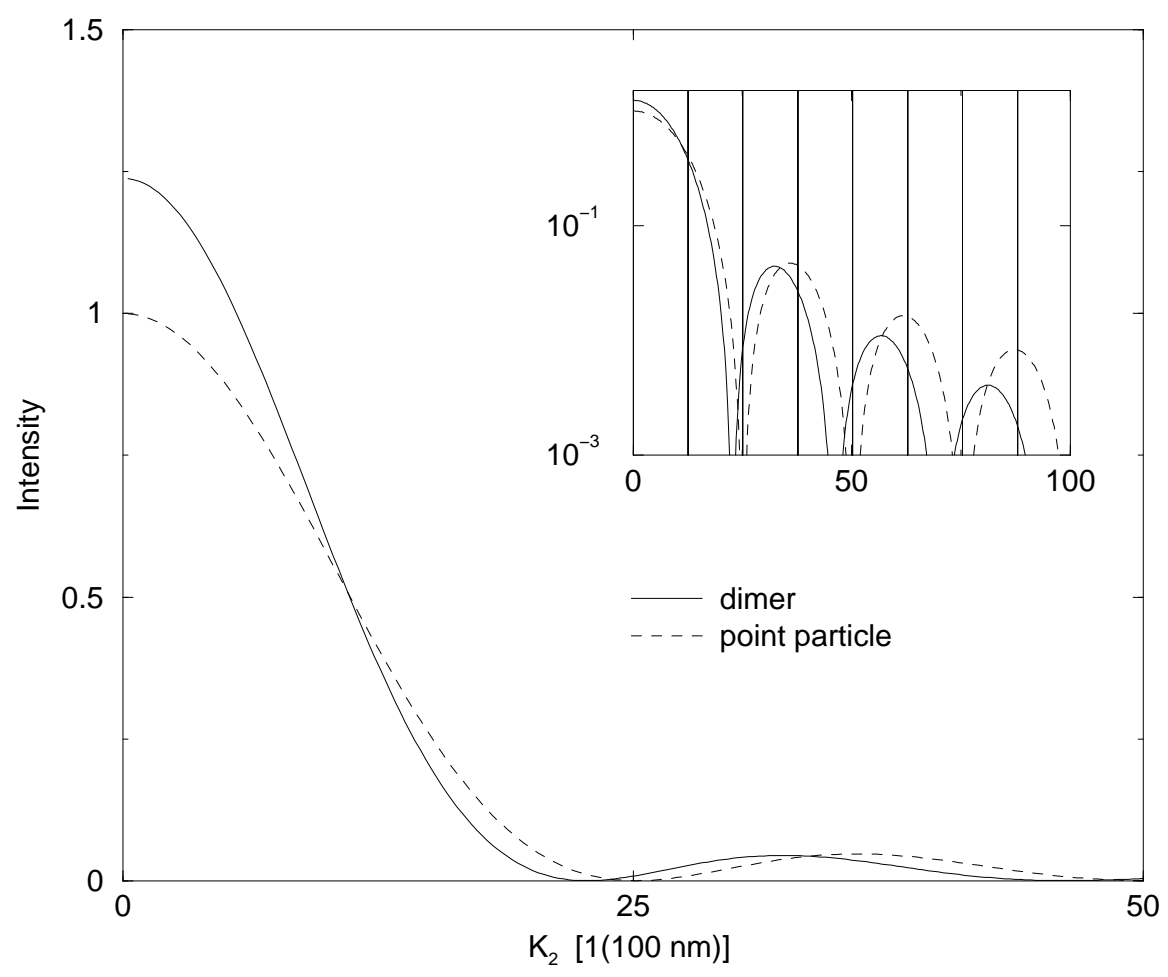

Figure 2: Diffraction by a single bar of width $25 \mathrm{~nm}$ for helium dimers and point particles. For a symmetric grating with this bar and slit width the vertical lines in the inset pick out the corresponding diffraction peaks. For dimers there are even order peaks which are absent for point particles. The lateral momentum transfer is $P_{2}=\hbar K_{2}$.

amplitude of $\mathrm{He}_{2}$, for a bar width of $25 \mathrm{~nm}$, can be approximated for small lateral momentum transfer by that of a point particle for bar width of $(25+2.8) \mathrm{nm}$. For a grating this would mean a nonsymmetric grating of correspondingly smaller slit width. The expectation value of $\left|x_{2}\right|$ for the ground-state wave-function of $\mathrm{He}_{2}$ we are using is just $2.8 \mathrm{~nm}$. A cumulant expansion of $t_{\mathrm{bar}}^{\mathrm{mol}}$ shows that the equality of these two numbers is not a coincidence [12].

\section{Location of the Diffraction Peaks}

Can one understand more directly and intuitively why the diffraction peaks occur at the same locations as for point particles of the same lateral momentum transfer? This is easy to see if one accepts that $t_{\text {incoh }}$ in Eq. (17) is negligible and that one just has to add the amplitudes from the individual bars. Indeed, the potential of the $n$th bar is the translate

$$
\exp \left\{-\mathrm{in} \hat{P}_{2} d / \hbar\right\} W_{\text {bar }} \exp \left\{\mathrm{in} \hat{P}_{2} d / \hbar\right\}
$$




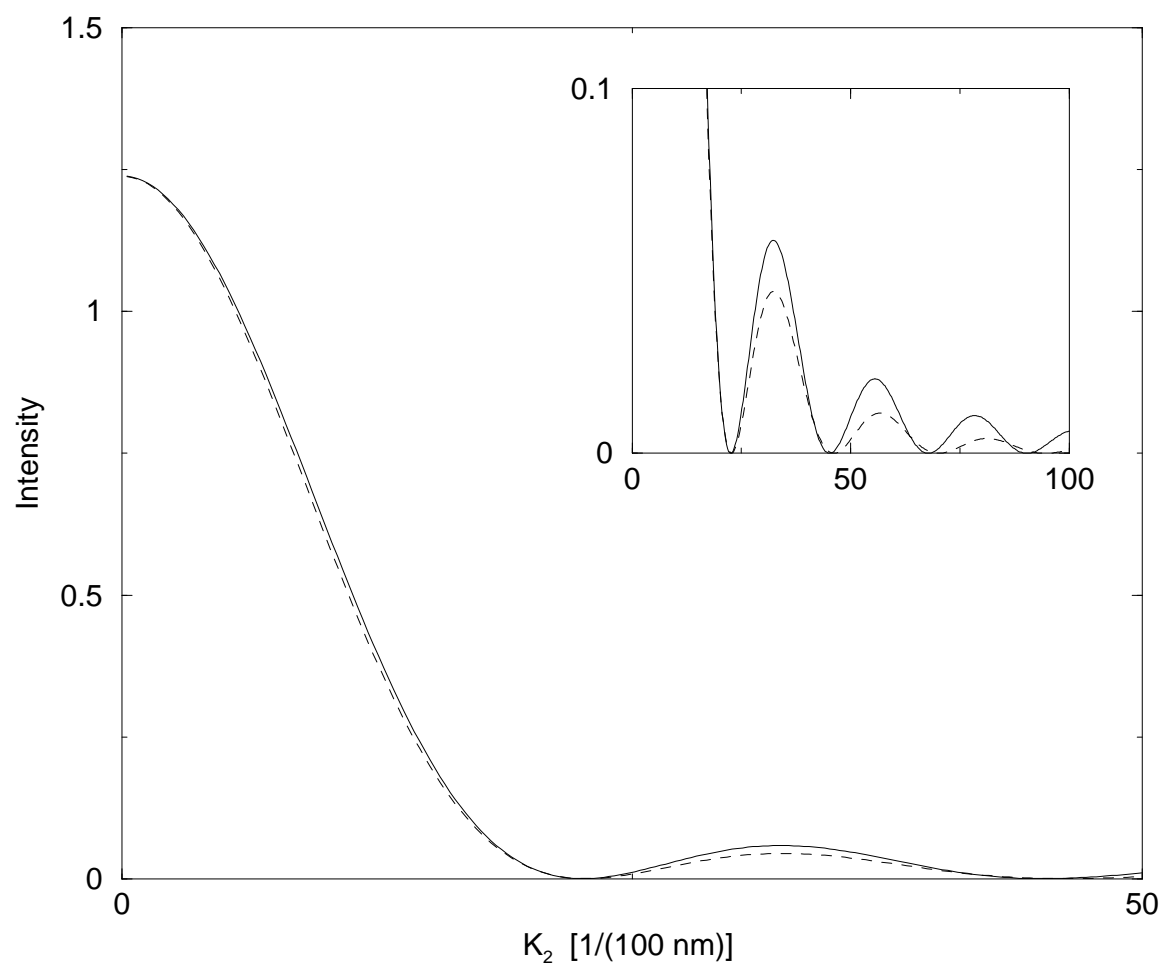

Figure 3: Diffraction of helium dimers (solid line) by a single bar of width $25 \mathrm{~nm}$ and diffraction of point particles (dashed line) by a single bar of width $(25+2.8)$ $\mathrm{nm}$. There is agreement for small lateral momentum transfer $P_{2}=\hbar K_{2}$.

of the first bar potential, and similarly for the $n$th bar transition amplitude $U_{V V}^{(n)}$. Since $\left|\mathbf{P}, \phi_{\gamma}\right\rangle$ is an eigenvector of the momentum operator $\hat{P}_{2}$, the associated amplitude differs just by the phase factor $\exp \left\{-\mathrm{i} n P_{2} d / \hbar\right\}$ from that of the first bar. Summation over $n$ leads to a sum over the phase factors, resulting in the grating function and Eq. (18).

There is also a symmetry argument for an infinite grating, exact to all orders and holding for larger clusters, too. For an infinite grating the complete Hamiltonian has period $d$ under translations in the 2 direction. Hence the Hamiltonian as well as the associated time-development operator $U(t)$ commute with $\exp \left\{-\mathrm{i} \hat{P}_{2} d / \hbar\right\}$. As a consequence

$$
\left\langle\mathbf{P}, \phi_{\gamma}|U(t)| \mathbf{P}^{\prime}, \phi_{\gamma^{\prime}}\right\rangle
$$

must vanish unless

$$
P_{2} / \hbar=\frac{2 \pi}{d} n, \quad n=0, \pm 1, \cdots
$$

and thus there are only transitions in these directions.

We recall that experimentally one always has a certain momentum spread and that only a part of the grating is illuminated by the incoming beam. To take this 
into account one can modify the above plane-wave formulation and it allows one to let $N$ become arbitrarily large [12]. The momentum spread then just leads to a broadening of the diffraction peaks. Incidentally, this procedure yields the correct height for the zeroth-order peak which otherwise would be misrepresented by a finite grating illuminated by an infinite plane wave. Figs. 4 and 5 show some results.

$$
\mathrm{d}=100 \mathrm{~nm}, \mathrm{~s}=50 \mathrm{~nm}
$$

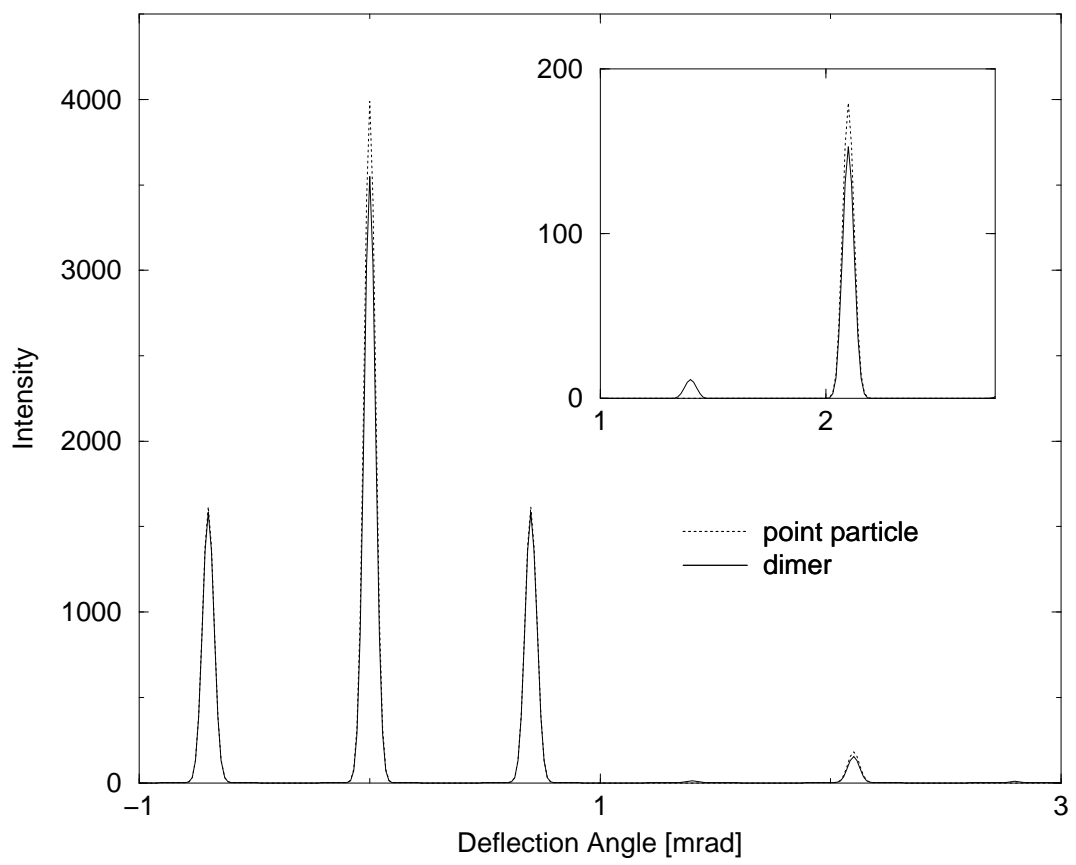

Figure 4: Diffraction by a symmetric grating with slit width $50 \mathrm{~nm}$ for helium dimers (solid line) and for corresponding point particles (dotted line). The differences are small, the second order peak barely visible.

\section{$5 \quad$ Further Developments}

Recent experiments by Schöllkopf and Toennies [13] have shown that an additional attractive potential may have an unexpectedly large influence on the diffraction patterns for higher noble atoms, increasing from neon to argon to krypton, in line with the increasing polarizability of these atoms. This attractive potential will also influence the diffraction pattern of bound systems to some extent.

In a first step we have taken this additional surface potential into account for diffraction of atoms. This can be done by using similar resolvent methods as in Section 2, with the surface potential treated as a perturbation. In the 
$\mathrm{d}=50 \mathrm{~nm}, \mathrm{~s}=25 \mathrm{~nm}$

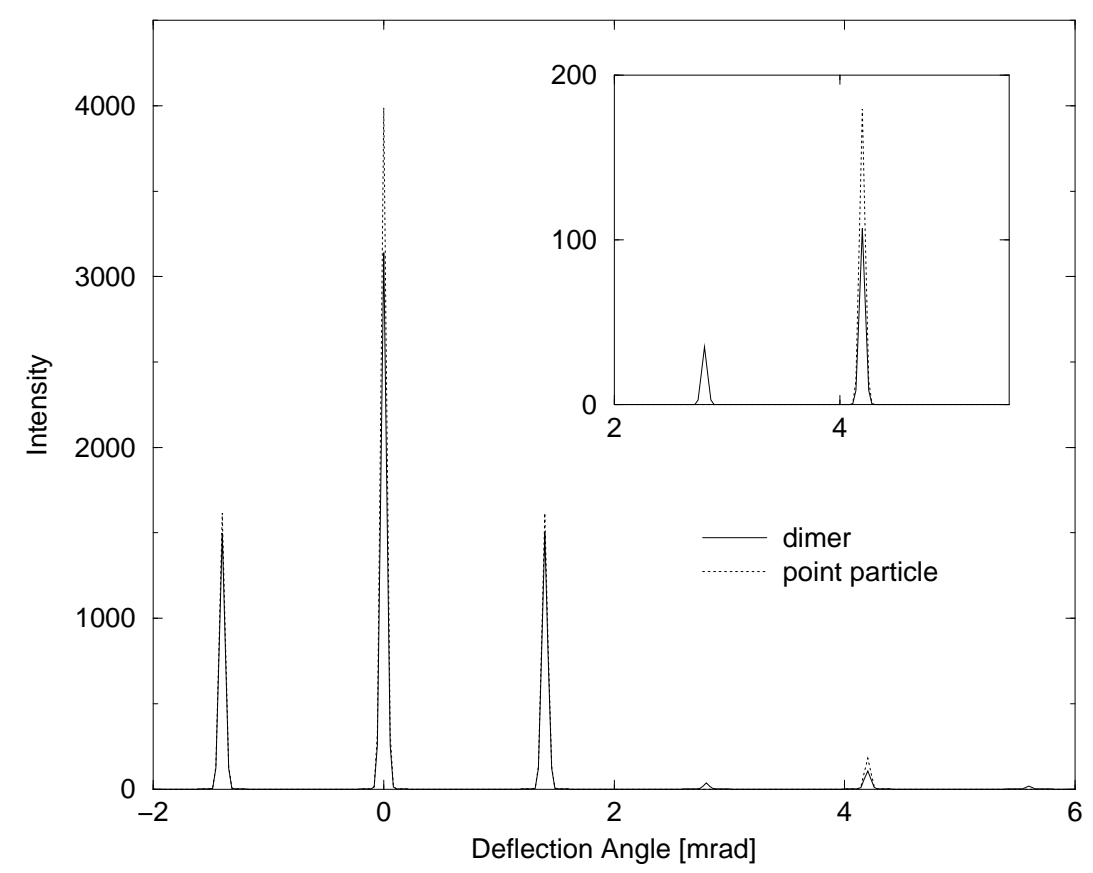

Figure 5: Diffraction by a symmetric grating with slit width $25 \mathrm{~nm}$ for helium dimers (solid line) and for point particles (dotted line). The differences are more pronounced, the second order peak is larger.

course of this something interesting happened. At first our results did not even qualitatively agree with the experiments for higher noble atoms - in particular the hierarchy of the peaks did not come out correctly - until we learned that due to the etching process the cross section of the grating bars was not rectangular as assumed by us but rather trapeze-like, with the angle off the perpendicular by $8^{0}$. Using this geometry agreement with the experiments was then obtained [12]. This, incidentally, shows the sensitivity of the theoretical methods.

The next step is to take the attractive surface potential into account for helium dimers. This work is presently in progress.

Another interesting topic is to use the cumulant expansion mentioned in the last section to obtain some measure of the 'size' of $\mathrm{He}_{2}$ from diffraction data and to obtain consequences for the binding potential.

In principle it should be possible to carry the approach of Section 2 over to three particles with external potential and apply it to the helium trimer, $\mathrm{He}_{3}$. In this connection a very interesting question is whether one can draw any conclusions from $\mathrm{He}_{3}$ diffraction patterns for the theoretically predicted Efimov state of $\mathrm{He}_{3}$ 14, 15. 


\section{Conclusions}

We have used few-body methods to investigate the diffraction of weakly bound two-particle systems by a transmission grating and have obtained explicit expressions for the transition amplitude in the elastic channel. The diffraction pattern is a product of the single-bar amplitude $t_{\mathrm{bar}}^{\mathrm{mol}}$ and the usual grating function. As for point particles our result depends only on $P_{2} / \hbar=(2 \pi / \lambda) \sin \varphi$ and on the bar width. The zeros of $t_{\mathrm{bar}}^{\mathrm{mol}}$ may differ from those for point particles so that even-order diffraction peaks may appear for symmetric gratings.

For large bar and slit widths $(\sim 10 \times$ bound-state diameter $)$ the diffraction pattern is still very close to that for point particles, although minor deviations exist (second order peak). For smaller bar and slit widths $(\sim 5 \times$ diameter $)$ significant deviations occur. The third order peak can be up to $40 \%$ lower. This can be attributed to breakups which should increase for larger momentum transfer. There is also an appreciable second order peak which is absent for point particles. This is more difficult to understand by breakups and is rather a finite-

size effect since the finite size can be partially taken into account by considering point particles and a nonsymmetric grating with smaller slit widths.

Additional attractive surface potentials which can, and do, modify the diffraction patterns can be taken into account.

Extension to helium trimers seems feasible, with a possible connection to Efimov states.

Acknowledgments: We would like to thank W. Schöllkopf and J. P. Toennies for letting us use some of their as yet unpublished results.

\section{References}

[1] O. Carnal and J. Mlynek, Phys. Rev. Lett. 66, 2689 (1991); D. W. Keith, C. R. Ekstrom, Q. A. Turchette, and D. E. Prichard, Phys. Rev. Lett. 66, 2693 (1991)

[2] W. Schöllkopf and J. P. Toennies, Science 266, 1345 (1994)

[3] M. S. Chapman, C. R. Ekstrom, T. D. Hammond, R. A. Rubenstein, J. Schmiedmayer, S. Wehinger, and D. E. Prichard, Phys. Rev. Lett. 74, 4783 (1995)

[4] For an overview of atom optics see the special issue Appl. Phys. B 54 (1992), edited by J. Mlynek, V. Balykin, and P. Meystre or C. S. Adams, M. Sigel, and J. Mlynek, Phys. Rep. 240, 143 (1994).

[5] W. Schöllkopf and J. P. Toennies, J. Chem. Phys. 104, 1155 (1996) 
[6] W. Schöllkopf and J. P. Toennies, to be published

[7] K. T. Tang, J. P. Toennies, and C. L. Yiu, Phys. Rev. Lett. 74, 1546 (1995)

[8] F. Luo, G. C. Mc Bane, G. Kim, C. F. Giese, and W. R. Gentry, J. Chem. Phys. 98, 3564 (1993)

[9] F. Luo, C. F. Giese, and W. R. Gentry, J. Chem. Phys. 104, 1151 (1995)

[10] G. C. Hegerfeldt and T. Köhler, Phys. Rev. A 57, 2021 (1998)

[11] E. O. Alt, P. Grassberger and W. Sandhas, Nucl. Phys. B2, 167 (1967)

[12] G. C. Hegerfeldt and T. Köhler, in preparation

[13] W. Schöllkopf and J. P. Toennies, private communication

[14] T. Cornelius and W. Glöckle, J. Chem. Phys. 85, 3906 (1986)

[15] B. D. Esry, C. D. Lin, and C. H. Green, Phys. Rev.A 54, 394 (1996) 